\title{
Dexamethasone Intravitreal Implant: An Effective Adjunctive Treatment for Recalcitrant Noninfectious Uveitis
}

\author{
E. Miserocchi G. Modorati M.R. Pastore F. Bandello \\ Department of Ophthalmology, University Vita-Salute, Scientific Institute San Raffaele, Milan, Italy
}

\section{Key Words}

Uveitis $\cdot$ Corticosteroids $\cdot$ Macular edema $\cdot$ Intravitreal dexamethasone

\begin{abstract}
Purpose: To describe our experience in treating recalcitrant and severe cases of noninfectious posterior uveitis with the 0.7-mg dexamethasone intravitreal implant as adjunctive anti-inflammatory treatment. Procedures: Retrospective study. Twelve patients ( 8 females; 4 males; 14 affected eyes, 15 implants) with severe recalcitrant uveitis were treated. Patients had an inadequate control of uveitis despite different immunosuppressants and periocular corticosteroid therapy. Primary outcome measures evaluated were: decrease in uveitis activity, improvement in visual acuity, reduction of macular thickness, and occurrence of adverse events. Results: Uveitis activity decreased in all patients after the implant. The mean follow-up time from injection was 9 months. Bestcorrected visual acuity improved from $20 / 80$ to $20 / 40$ at the end of follow-up. The mean retinal thickness improved from 496 to $226 \mu \mathrm{m}$. Adverse events encountered were: 3 eyes with intraocular pressure elevation, 1 vitreous hemorrhage and 1 subconjunctival hemorrhage. Three patients reduced the daily systemic corticosteroid dosage after treatment.
\end{abstract}

Conclusions: The $0.7-\mathrm{mg}$ dexamethasone intravitreal implant appears to be a novel and promising adjunctive treatment for patients with severe posterior noninfectious uveitis recalcitrant to different immunosuppressive agents.

Copyright $\odot 2012$ S. Karger AG, Basel

\section{Introduction}

Uveitis encompasses a group of potentially blinding inflammatory diseases that are responsible for $2.8-10 \%$ of all cases of blindness. The importance of this sight-threatening disease is translated in numbers. Studies have estimated that uveitis may lead to legal blindness in 30,000 patients annually $[1,2]$. Patients with uveitis are in desperate need of effective therapeutic agents which not only eliminate inflammation and prevent recurrences but also protect the patients from potential side effects. During the last decade, the therapeutic armamentarium of the uveitis specialists has enormously expanded. Novel biologic agents and intraocular therapy are becoming a valuable alternative treatment to control ocular inflammatory diseases and inflammatory macular edema, the most important cause of visual loss in patients with uveitis $[3,4]$.

\section{KARGER}

Fax +4161306 1234

E-Mail karger@karger.ch

www.karger.com
(C) 2012 S. Karger AG, Basel

$0030-3755 / 12 / 2284-0229 \$ 38.00 / 0$

Accessible online at:

www.karger.com/oph
Elisabetta Miserocchi

Department of Ophthalmology, Scientific Institute San Raffaele

University Vita-Salute

Via Olgettina 60, IT-20132 Milan (Italy)

E-Mail miserocchi.elisabetta@hsr.it 
Despite the large variety of new drugs available to treat uveitis, corticosteroids are still the gold standard of treatment of noninfectious uveitis. Corticosteroid drugs are inexpensive, fast acting, and potent, properties that have made them the first line of therapy for virtually all patients with noninfectious ocular inflammatory disease [5-8].

However, the different available types of corticosteroids delivery can carry significant ocular and systemic side effects which can limit their use in the treatment of uveitis. In an effort to avoid the side effects associated with systemic steroids, sustained-release intraocular corticosteroid implants have been developed $[9,10]$. The dexamethasone drug delivery system (Ozurdex ${ }^{\circledR}$; Allergan Inc., Irvine, Calif., USA) is a biodegradable intravitreal implant with potent anti-inflammatory properties, and a favorable side effect profile. Recent studies have demonstrated that this implant is able to improve visual acuity and macular thickness in different ocular conditions such as macular edema associated with retinal vein occlusion [11, 12] and uveitis [13-15].

The purpose of the present study is to describe our experience in treating recalcitrant and severe cases of noninfectious posterior uveitis with the $0.7-\mathrm{mg}$ dexamethasone intravitreal implant as adjunctive anti-inflammatory treatment.

\section{Methods}

We conducted a retrospective analysis of the case records of 12 patients with chronic posterior noninfectious uveitis who received dexamethasone intravitreal implant from August 2011 to June 2012.

The study was conducted in compliance with the tenets of the Declaration of Helsinki and written informed consent was obtained from each patient.

All patients attended the Ocular Immunology and Uveitis Service at the San Raffaele Scientific Institute in Milan, where they were always under the care of the same uveitis specialist (E.M.). Patients included in the study were those who had an inadequate response in controlling intraocular inflammation with recurrence of posterior uveitis and macular edema despite different immunosuppressants (conventional and biologics) and periocular corticosteroid therapy.

Patients underwent a complete ophthalmologic assessment including best-corrected visual acuity, slit-lamp biomicroscopy, intraocular pressure (IOP), ophthalmoscopy and spectral domain optical coherence tomography at each visit. Fluorescein angiography was performed at baseline and when needed during followup visits.

Ophthalmic assessment after intraocular injection was performed at 1 week and then monthly according to disease activity and response to treatment. Data collected included: demograph- ics, duration of uveitis, characteristics of the uveitis (etiology and anatomic classification), associated systemic disease, best-corrected visual acuity, central macular thickness measured using optical coherence tomography, ocular complications, systemic immunosuppressant therapies, follow-up, and adverse events.

The primary outcome measures evaluated were: decrease in uveitis activity (vitreous haze), improvement in visual acuity, reduction of macular thickness, reduction of concomitant systemic corticosteroid and/or immunosuppressants, and occurrence of adverse events. Disease activity was graded in accordance with the Standardization of Uveitis Nomenclature (SUN) criteria [16].

The dexamethasone intravitreal implant was inserted into the vitreous cavity through the pars plana using a customized, singleuse, 22-gauge applicator device under topical anesthesia. Patients were treated with a topical ophthalmic antibiotic 4 times daily starting 3 days before the procedure and continuing for 7 days after the injection.

\section{Results}

A total of 12 Caucasian patients ( 8 females; 4 males; 14 affected eyes, 15 implants) with uveitis were treated with the 0.7-mg dexamethasone intravitreal implant.

Demographic characteristics of patients are summarized in table 1 . The mean $( \pm S D)$ age of patients was $55,16 \pm 15$ years (range $32-82$ ) and the mean follow-up time from injection was 9 months (range 6-11). The uveitis etiology was: idiopathic $(\mathrm{n}=4)$, Behçet's disease $(\mathrm{n}=$ $2)$, sarcoidosis $(n=2)$, rheumatoid arthritis $(n=2)$, HLAB27 $(\mathrm{n}=1)$ and multiple sclerosis $(\mathrm{n}=1)$.

Indication for treatment with dexamethasone intravitreal implant was macular edema in 10 , active retinal vasculitis in 2, vitritis in 3 and active choroiditis in 2 patients. In patient No. 3 with serpiginous choroiditis, indication for implant was the need for reduction of systemic corticosteroid therapy due to important steroid-related side effects (severe hypertension and pathologic bone fracture). All patients received one dexamethasone implant, while 2 patients received more than one injection in both eyes.

Table 2 shows the results of visual acuity, follow-up, associated systemic treatment before and after the treatment and the occurrence of adverse events.

The mean best-corrected visual acuity before the implant was 20/80 and the mean postoperative visual acuity at the end of follow-up was 20/40 in the treated eyes. The mean preoperative retinal thickness measured by optical coherence tomography was $496 \pm 123 \mu \mathrm{m}$ (range 320682) and the mean value at the end of follow-up was 226 $\pm 66 \mu \mathrm{m}$ (range 160-331). 
Table 1. Demographic characteristics of patients

\begin{tabular}{|c|c|c|c|c|c|c|}
\hline $\begin{array}{l}\mathrm{Pa}- \\
\text { tient }\end{array}$ & Sex & Age & Diagnosis & Location & $\begin{array}{l}\text { Dexamethasone } \\
\text { implant }\end{array}$ & Indication for treatment \\
\hline 1 & M & 47 & HLA-B27 & Posterior & Right eye; left eye & Cystoid macular edema, vitritis \\
\hline \multirow[t]{2}{*}{3} & M & 62 & Serpiginous choroiditis & Posterior & Right eye 2 ; left eye & Active choroiditis \\
\hline & & & & & & Reduction systemic steroids \\
\hline 4 & $\mathrm{~F}$ & 73 & Idiopathic & Intermediate & Left eye & Cystoid macular edema \\
\hline 6 & $\mathrm{~F}$ & 57 & Idiopathic multifocal choroiditis & Posterior & Right eye & Active choroiditis \\
\hline 7 & $\mathrm{~F}$ & 76 & Rheumatoid arthritis & Posterior & Left eye & Cystoid macular edema \\
\hline 8 & $\mathrm{M}$ & 52 & Sarcoidosis & Posterior & Right eye & Cystoid macular edema, vitritis \\
\hline 9 & $\mathrm{~F}$ & 45 & Idiopathic & Intermediate & Right eye & Cystoid macular edema \\
\hline 10 & M & 46 & Behçet & Posterior & Left eye & Cystoid macular edema, retinal vasculitis \\
\hline 11 & $\mathrm{~F}$ & 37 & Sarcoidosis & Posterior & Left eye & Cystoid macular edema, vitritis \\
\hline
\end{tabular}

Table 2. Visual acuity, follow-up, systemic treatment and adverse events of patients

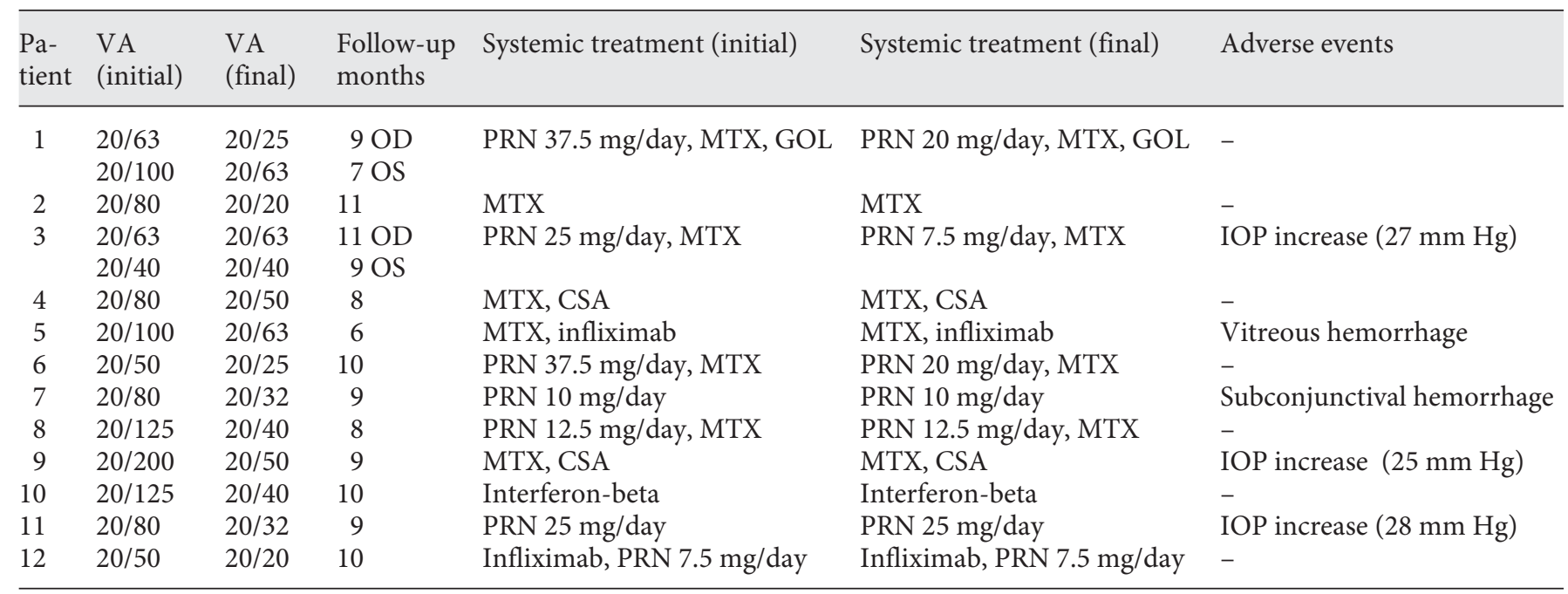

$\mathrm{VA}=$ Visual acuity; $\mathrm{OD}=$ right eye $\mathrm{OS}=$ left eye; $\mathrm{PRN}=$ prednisolone; $\mathrm{GOL}=$ golimumab; $\mathrm{MTX}=$ methotrexate $\mathrm{CSA}=\mathrm{cyclosporine}$.

Transient IOP elevation (lower than $30 \mathrm{~mm} \mathrm{Hg}$ ) was seen in 3 patients after the implant; the IOP increase was noticeable after 2 weeks from the injection and was controlled with topical antiglaucoma treatment (combined beta-blockers and dorzolamide drops twice a day) in all patients. The major adverse event encountered in our treated eyes was vitreous hemorrhage 1 day after the injection in a patient who was on anticoagulant treatment; the vitreous cleared up after 1 month with visual acuity improvement and resolution of macular edema. Another patient had a minor painless subconjunctival hemorrhage that resolved spontaneously 1 week after the surgical procedure.

All our patients were on systemic immunosuppressive therapy and corticosteroids for the associated autoimmune disease or for the long-standing recalcitrant uveitis, before receiving the implant. Three patients were able to reduce the daily corticosteroid dosage after the dexamethasone intravitreal implant. 


\section{Discussion}

The present study showed that the $0.7-\mathrm{mg}$ dexamethasone intravitreal implant may be an effective adjunctive treatment in patients with long-standing uveitis refractory to conventional anti-inflammatory treatment. Before the intravitreal injection, our patients were treated with different systemic corticosteroids and/or immunosuppressants and periocular steroid injections. Despite these different treatment strategies, our patients have experienced recurrences of intraocular inflammation in the form of macular edema, vitritis or retinal vasculitis and were offered to receive the $0.7-\mathrm{mg}$ dexamethasone intravitreal implant as adjunctive treatment.

All patients showed reduction of the uveitis activity and visual acuity improvement after the dexamethasone intravitreal implant. Among the 14 treated eyes, there was a mean improvement from baseline of the best-corrected visual acuity of 3.3 lines at the end of follow-up (range 0-6 lines).

We observed a decrease from baseline central macular thickness on ocular coherence tomography in our patients with macular edema; the reduction of macular thickness was extremely rapid and noticeable around the second week after the injection. These data are in accordance with other series present in the literature [13-15]. Three of our patients (25\%) developed moderate IOP increase after dexamethasone intravitreal implant and required combined antiglaucoma therapy. In a large clinical trial of 229 patients with uveitis, randomized to receive either sham procedure or treatment with dexamethasone implant, and observed for 26 weeks, Lowder et al. [13] showed that $10 \%$ of eyes had an IOP of $25 \mathrm{~mm} \mathrm{Hg}$ or greater and throughout the duration of the study $23 \%$ of eyes in the $0.7-\mathrm{mg}$ dexamethasone implant group required IOP-lowering medications. In our treated patients, no eyes required surgical or laser therapy for elevated IOP, similarly to the results of other larger studies in the literature $[13,17]$. Cataract formation was not observed in our small sample of patients, although this complication is reported in about $15 \%$ of eyes treated with the dexamethasone intravitreal implant [11-15].

All our patients maintained the systemic immunosuppressive therapy after the dexamethasone implant; however, in 3 patients, we were able to taper systemic corticosteroids after the injections. In patient No. 3 with serpiginous choroiditis, who received 3 consecutive implants in both eyes, the main indication for implant was reduction of systemic corticosteroids due to important steroid-related side effects.
The major adverse event that we observed in our group of patients was vitreous hemorrhage in patient No. 5 who was on systemic anticoagulation for chronic atrial fibrillation. The macular edema and the visual acuity in this patient improved after 1 month with complete resolution of vitreous hemorrhage.

In the present study, we showed that the intravitreal dexamethasone implant can be an effective adjunctive treatment option to reduce intraocular inflammation in patients with recalcitrant uveitis already on systemic immunosuppression.

In patients with recurrence of posterior uveitis and important visual loss that require a rapid resolution of vitritis or macular edema, the intravitreal corticosteroid implant may be an effective therapeutic alternative to the increase of systemic immunosuppressive therapy.

In conclusion, the dexamethasone drug delivery system appears to be a novel and promising approach to the treatment of recalcitrant uveitis and has been shown to be effective in the treatment of noninfectious uveitis with potentially fewer adverse effects than other steroid treatment modalities. However, careful selection of patients is always warranted to avoid complications, in particular exclusion of infectious forms of uveitis and masquerade syndromes, exclusion of patients with prior IOP elevation or known history of glaucoma, and the risk-benefit assessment for discontinuation of systemic anticoagulation to avoid vitreous hemorrhages.

Future studies will need to determine the relative efficacy and safety profiles among the different types of uveitis and to assess the long-term effects of repeated use of the dexamethasone implant in the treatment of recurrent uveitis.

References

1 Suttorp-Schulten MS, Rothova A: The possible impact of uveitis in blindness: a literature survey. Br J Ophthalmol 1996;80:844848.

2 Nussenblatt RB: The natural history of uveitis. Int Ophthalmol 1990;14:303-308.

3 Miserocchi E, Modorati G, Foster CS: New treatments in noninfectious uveitis; in Bandello F (eds): Developments in Ophthalmology. Basel, Karger, vol 51, pp 134-161.

4 Larson T, Nussenblatt RB, Sen HN: Emerging drugs for uveitis. Expert Opin Emerg Drugs 2011;16:309-322.

5 Sherif Z, Pleyer U: Corticosteroids in ophthalmology: past-present-future. Ophthalmologica 2002;216:305-315.

-6 Taylor SR, Isa H, Joshi L, Lightman S: New developments in corticosteroid therapy for uveitis. Ophthalmologica 2010;224:46-53. 
7 Cunningham ET Jr, Wender JD: Practical approach to the use of corticosteroids in patients with uveitis. Can J Ophthalmol 2010; 45:352-358.

$>8$ Edelman JL: Differentiating intraocular glucocorticoids. Ophthalmologica 2010;224: 25-30.

$>9$ Callanan DG, Jaffe GJ, Martin DF, Pearson PA, Comstock TL: Treatment of posterior uveitis with a fluocinolone acetonide implant: three-year clinical trial results. Arch Ophthalmol 2008;126:1191-1201.

10 Ghosn CR, Li Y, Orilla WC, Lin T, Wheeler L, Burke JA, Robinson MR, Whitcup SM: Treatment of experimental anterior and intermediate uveitis by a dexamethasone intravitreal implant. Invest Ophthalmol Vis Sci 2011;52:2917-2923.
11 Haller JA, Bandello F, Belfort R Jr, Blumenkranz MS, Gillies M, Heier J, Loewenstein A, Yoon YH, Jiao J, Li XY, Whitcup SM: Dexamethasone intravitreal implant in patients with macular edema related to branch or central retinal vein occlusion twelve-month study results. Ophthalmology 2011;118: 2453-2460.

12 Yeh WS, Haller JA, Lanzetta P, Kuppermann BD, Wong TY, Mitchell P, Whitcup SM, Kowalski JW: Effect of the duration of macular edema on clinical outcomes in retinal vein occlusion treated with dexamethasone intravitreal implant. Ophthalmology 2012; 119:1190-1198.

13 Lowder C, Belfort R Jr, Lightman S, Foster CS, Robinson MR, Schiffman RM, Li XY, Cui H, Whitcup SM: Dexamethasone intravitreal implant for noninfectious intermediate or posterior uveitis. Arch Ophthalmol 2011;129:545-553.
14 Myung JS, Aaker GD, Kiss S: Treatment of noninfectious posterior uveitis with dexamethasone intravitreal implant. Clin Ophthalmol 2010;4:1423-1426.

15 Williams GA, Haller JA, Kuppermann BD, Blumenkranz MS, Weinberg DV, Chou C, Whitcup SM: Dexamethasone posteriorsegment drug delivery system in the treatment of macular edema resulting from uveitis or Irvine-Gass syndrome. Am J Ophthalmol 2009; 147:1048-1054.

16 Jabs DA, Nussenblatt RB, Rosenbaum JT, Standardization of Uveitis Nomenclature (SUN) Working Group: Standardization of uveitis nomenclature for reporting clinical data. Results of the First International Workshop. Am J Ophthalmol 2005;140:509-516.

17 Saraiya NV, Patel SS, Goldstein DA: A report of high intraocular pressure with the dexamethasone intravitreal implant. Arch Ophthalmol 2011;129:1638-1639. 